\title{
Análisis de una intervención educativa para el desarrollo de la autodeterminación en una alumna con síndrome de Down
}

Carla Moscoso José - Universitat de València Irene Lacruz Pérez - Universitat de València Raúl Tárraga Mínguez - Universitat de València
0000-0003-3153-3056

D 0000-0003-1560-1952

0000-0002-4458-5763

Recepción: 01.10.2020 | Aceptado: 03.02.2021

Correspondencia a través de ORCID: Raúl Tárraga Mínguez

iD 0000-0002-4458-5763

Citar: Moscoso José, C., Lacruz Pérez, I. y Tárraga Mínguez, R. (2021). Análisis de una intervención educativa para el desarrollo de la autodeterminación en una alumna con síndrome de Down. REIDOCREA, 10(5), 1-11. [ ]

Resumen: Antecedentes: uno de los mayores problemas a los que se enfrentan los jóvenes con discapacidad intelectual es su bajo nivel de autonomía. Objetivo: el objetivo de este trabajo es analizar la eficacia de una intervención basada en el uso de las tecnologías de la información y comunicación (TIC) para alcanzar mejoras en autodeterminación en una estudiante con síndrome de Down. Método: la intervención está basada en el diseño y aplicación de una página web diseñada teniendo en cuenta las necesidades específicas de la participante, su nivel de competencias, y sus intereses. Esta herramienta TIC permite a la estudiante trabajar de manera autónoma tres áreas de conocimiento distintas y otros aspectos de la vida diaria como las tareas del hogar o el comportamiento en lugares públicos. Como instrumento de evaluación, se ha utilizado la escala ARC-INICO. Conclusiones: Ios resultados muestran un desarrollo notable en los niveles globales de autodeterminación de la joven, especialmente en la dimensión de autonomía. Este resultado muestra que el uso de las TIC puede acarrear numerosos beneficios a las personas con diversidad funcional, lo cual debería tenerse en cuenta en el ámbito educativo puesto que podría facilitar la inclusión del alumnado con necesidades educativas especiales (NEE).

Palabra clave: Discapacidad intelectual

\section{Analysis of an educational intervention to develop self-determination in a student with Down} syndrome

Abstract: Introduction: one of the biggest problems that young people with intellectual disabilities face is their low level of autonomy. Aim: The aim of this work is to analyze the efficacy of an intervention based on the use of information and communication technologies (ICT) to achieve improvements in self-determination in a student with Down syndrome. Methods: the intervention is based on the design and application of a web page designed taking into account the specific needs of the participant, her level of skills, and her interests. This ICT tool allows the student to work autonomously in three different areas of knowledge and other aspects of daily life such as housework or behavior in public places. The ARC-INICO scale has been used as assess instrument. Conclusions: the results show a remarkable development in the global levels of selfdetermination of the student, especially in the dimension of autonomy. This result shows that the use of ICT can bring numerous benefits to people with functional diversity, which should be taken into account in the educational field since it could facilitate the inclusion of students with special educational needs (SEN).

Keyword: Intellectual disability

\section{Introducción}

Las tecnologías de la información y la comunicación (TIC) cumplen un papel importante en la inclusión social y educativa de las personas con diversidad funcional al ofrecerles la oportunidad de mejorar su calidad de vida proporcionándoles, entre otros aspectos, una mayor autonomía. En el ámbito educativo son herramientas que permiten dar respuesta a las necesidades educativas especiales (NEE) de algunos estudiantes, contribuyendo así a formar un sistema educativo más inclusivo.

En el caso concreto de las personas con discapacidad intelectual, este trastorno del neurodesarrollo les puede suponer limitaciones tanto en su funcionamiento intelectual 
como en su comportamiento adaptativo en los ámbitos conceptual, social y práctico (American Psychiatric Association, 2013). Esto deriva en que las personas con discapacidad intelectual se encuentren frecuentemente en situación de dependencia, es decir, que precisen ayuda para realizar actividades de la vida diaria o incluso apoyos para su autonomía personal.

El grado de dependencia de la persona con discapacidad intelectual se ve influido por su nivel de autodeterminación, siendo este uno de los indicadores más relevantes de calidad de vida de las personas (Claes, Van Hove, Vandevelde, Van Loon y Schalock, 2012; Peralta y Arellano, 2014).

Existen hasta cuatro modelos teóricos diferentes para definir el concepto de autodeterminación, pero el modelo funcional de Michael Wehmeyer es el que se ha difundido ampliamente en España y ha fundamentado numerosas investigaciones en nuestro país. De acuerdo a este modelo, la autodeterminación es el conjunto de acciones que capacitan a la persona para actuar como agente primario de su propia vida y para mejorar la calidad de la misma (Peralta y Arellano, 2014).

Dada su importancia, el desarrollo de la autodeterminación debe ser un objetivo primordial en el proceso educativo de las personas con discapacidad intelectual, sobre todo teniendo en cuenta que las familias tienden a limitar la toma de decisiones de sus hijos al creer que no están capacitados para ello (Peralta y Arellano, 2014).

Para educar en autodeterminación se requiere un cambio importante en la manera de planificar los apoyos escolares, familiares y profesionales del proceso de aprendizaje del alumno, lo cual puede realizarse a través de las TIC. En el ámbito educativo esto exige que el profesorado esté motivado, implicado y formado adecuadamente en el manejo de las tecnologías (Fernández, Reyes y El Homran, 2018).

Pese a que las publicaciones en torno a este tema no son abundantes, existe evidencia empírica de que mediante el uso de las TIC se pueden mejorar determinadas habilidades de las personas con diversidad funcional.

Cullen y Alber-Morgan (2015) llevaron a cabo una revisión teórica de 36 estudios que analizaban los efectos del uso de las tecnologías en las habilidades de las personas con discapacidad intelectual principalmente para realizar tareas de la vida diaria de forma independiente. En general, la mayoría de estudios apuntaron resultados positivos, y los autores resaltaron como dos de los factores más positivos de las TIC el hecho de que estos recursos puedan personalizarse para cada usuario; y el efecto positivo en la motivación que se produce en los estudiantes con discapacidad intelectual al utilizar los mismos recursos TIC que otros compañeros de su misma edad sin discapacidad intelectual.

Ahondando en esta misma línea, Draper, Strnadová y Cumming (2014) realizaron una revisión de estudios sobre el uso de iPads en educación especial desde diferentes perspectivas: la de los estudiantes, los docentes y los familiares. Los resultados mostraron que tanto los padres como el profesorado valoraban positivamente el uso del iPad como herramienta educativa, puesto que ofrece la oportunidad de trabajar con mayor independencia, repercute favorablemente en el comportamiento del niño y su autodeterminación y facilita la adquisición de conocimientos y habilidades. Respecto a los estudiantes, fueron significativamente más comprometidos en las actividades académicas cuando usaban las aplicaciones. Por consiguiente, Draper et al. (2014), aseguran que la integración de la tecnología de apoyo en el aula inclusiva promueve el acceso, la participación y el progreso de los estudiantes con discapacidad intelectual. 
Además de estas revisiones, recientemente también se han llevado a cabo estudios que han analizado de manera específica el efecto de determinados recursos TIC en los niveles de autodeterminación de personas con discapacidad intelectual. Por ejemplo, en el estudio de Cannella-Malone, Konrad y Pennington (2015) se pretendía proporcionar a los maestros herramientas tecnológicas para trabajar la expresión escrita con alumnado con discapacidad intelectual. Los resultados de este estudio evidenciaron que los niveles de autodeterminación y motivación de los estudiantes habían aumentado considerablemente tras el uso de determinadas tecnologías de asistencia. Por último, el estudio de Stasolla et al. (2014) evaluó el éxito de los soportes tecnológicos en dos niños con síndrome de $X$ frágil con profundo retraso de desarrollo. En base a los resultados, Stasolla et al. (2014) concluyeron que las TIC son útiles en el proceso de desarrollo de los usuarios, mostrando un crecimiento de los índices de felicidad y una disminución de los comportamientos estereotipados, además de constituir una importante forma de apoyo a los padres en el proceso.

En definitiva, la mayoría de investigaciones coinciden en afirmar que las TIC, teniendo en cuenta los beneficios que suponen para las personas con diversidad funcional, son actualmente una de las herramientas más valiosas para mejorar la calidad de vida de estas personas, en especial en lo que se refiere a su autonomía e independencia.

\section{Objetivos}

El objetivo de este estudio es diseñar una página web como herramienta para desarrollar la autodeterminación y la autonomía en una alumna con síndrome de Down. Consideramos que este objetivo puede extender las conclusiones obtenidas en las investigaciones previas, en tanto que aportará datos sobre la capacidad de las TIC para mejorar los niveles de autodeterminación en un contexto cercano, y utilizando un instrumento de evaluación riguroso específicamente encaminado a evaluar este constructo de autodeterminación.

\section{Métodos}

\section{Participante}

En esta investigación ha participado una adolescente de 17 años con síndrome de Down, su padre, su madre y ocasionalmente la tutora de la fundación a la que asiste. La participante está escolarizada en un centro ordinario de la ciudad de Valencia y cursa los estudios de $4^{\circ}$ de Educación Secundaria Obligatoria (ESO). No obstante, la prueba de Nivel Actual de Competencias (N.A.C), que le fue administrada en su centro educativo, sitúa a la participante en un nivel cognitivo correspondiente a $3^{\circ}$ de educación primaria.

En su centro educativo trabaja con el especialista de pedagogía terapéutica (PT) un día a la semana y con el de audición y lenguaje $(A L)$ dos sesiones semanales. Fuera del centro educativo asiste tres veces por semana a una fundación especializada en el trabajo con personas con síndrome de Down, en la que realiza tanto sesiones educativas como actividades de ocio.

Cuenta con un buen ambiente familiar: su hermano mayor es un gran apoyo para ella y sus padres están implicados de forma directa en el proceso educativo de su hija y son activos en la defensa de los derechos de las personas con síndrome de Down y sus familias. 


\section{Instrumento}

Para conocer los niveles de autodeterminación de la estudiante se empleó la escala ARC-INICO (Verdugo et al., 2014). Esta escala se basa en el modelo funcional de autodeterminación de Wehmeyer, y tiene como objetivo evaluar las diferentes dimensiones del comportamiento y el nivel global de autodeterminación en jóvenes de entre 11 y 19 años con discapacidad intelectual o del desarrollo.

Las características psicométricas de esta escala la convierten en una fuente fiable y válida. Está compuesta por 61 ítems divididos en cuatro secciones de evaluación: autonomía, autorregulación, empoderamiento y autoconocimiento.

Esta prueba se administró antes de la intervención (pretest) con el fin de diseñar un programa ajustado a las necesidades individuales y específicas de la alumna, y después (posttest), para valorar la eficacia del programa de intervención y su impacto sobre la autodeterminación de la usuaria.

Además, en este estudio se llevaron a cabo entrevistas informales con la familia y la tutora de la fundación tanto al inicio como a lo largo de todo el proceso (en forma de evaluación inicial y continua).

\section{Procedimiento}

El proceso global de este estudio podría dividirse en cinco fases. La primera de ellas fue conocer a la alumna y sus necesidades.

Tras solicitar los permisos pertinentes a la dirección de la fundación, a la tutora y a su familia, se llevó a cabo la recogida de información mediante reuniones y entrevistas. Dicha información se refería principalmente a aspectos como el ritmo de aprendizaje de la estudiante, su nivel de competencia, el tipo de actividades en el que trabaja, la disponibilidad de un ordenador personal en casa, su conocimiento tecnológico previo o las limitaciones que encuentra en las actividades diarias.

La segunda fase del procedimiento fue la aplicación de una prueba pretest para evaluar las dimensiones de autodeterminación de la alumna mediante la escala ARC-INICO (Verdugo et al., 2014). Se administró por primera vez en el mes de octubre de 2019, tanto a la estudiante como a sus familiares para así poder comparar ambas perspectivas.

En el caso de la alumna, el evaluador únicamente actuó como colaborador siendo ella quien guió el proceso de evaluación, que tuvo lugar en su aula habitual de la fundación. A la familia se le entregó una copia del cuestionario junto con la explicación teórica necesaria para que lo pudieran cumplimentar correctamente en su domicilio, y las respuestas fueron respondidas conjuntamente y consensuadas por el padre y la madre.

La tercera fase del estudio consistió en el diseño de una intervención cuyo objetivo general fue fomentar la competencia tecnológica para favorecer el desarrollo de la autonomía en la participante. Este objetivo general se concretaba en los siguientes objetivos específicos: fomentar la voluntad e iniciativa de trabajar y aprender; hacer un uso adecuado de las TIC; adquirir las competencias educativas planteadas; aprender autónomamente; utilizar productos de limpieza básicos; aprender a realizar tareas del hogar; utilizar una agenda personal para organizarse en el tiempo; conocer las normas de convivencia y las pautas para desenvolverse en lugares públicos; y, por último, 
aprender a elaborar recetas y a utilizar utensilios de cocina adecuados para su nivel cognitivo.

Se elaboró una página web con contenido y herramientas destinadas al desarrollo del nivel de autodeterminación de la alumna. Para ello, se utilizó la plataforma Wix.com, que permite crear y diseñar sitios web HTML5 a través del uso de herramientas sencillas y prácticas. Asimismo, ofrece la posibilidad de editar y publicar las ediciones al instante, lo que permite modificar y adaptar el proceso de intervención al usuario.

Durante el proceso de diseño se tuvieron en cuenta los gustos y preferencias de la participante para que así la herramienta le resultara atractiva y motivadora. A modo de ejemplo, el título de la página web, Aprende con el corazón, se sitúa en el encabezado con tonos azulados puesto que este es el color favorito de la alumna.

La página web consta de cuatro secciones claramente diferenciadas: Inicio, Actividades, Mi agenda y Guías. En todas ellas existe una herramienta permanente a través de la cual la usuaria puede consultar sus dudas, que serán transmitidas a la persona administradora de la página en forma de correo electrónico.

La sección Inicio incluye una galería de imágenes con transición, basadas en sus gustos sobre animales, paisajes y aficiones; un mensaje motivador; y un reproductor de música con diferentes canciones de su gusto (no es accesible cuando se están realizando las actividades).

Teniendo en cuenta los resultados de la prueba N.A.C, la sección de Actividades, se divide en lengua castellana, matemáticas y ciencias de la naturaleza, ya que fueron las áreas en las que la alumna tenía menor nivel.

En cada materia las actividades están divididas por contenidos y se accede a ellas mediante enlaces directos que, en algunos casos, redirigen a un documento donde la alumna puede escribir las respuestas (se guardan de forma automática y pueden ser corregidas por ella misma), y en otros casos a juegos educativos para aprender interactivamente.

Las actividades están diseñadas en función de los objetivos planteados en el Plan de Trabajo Individual (PTI) de la estudiante. En el área de lengua castellana se trabaja comprensión lectora, expresión escrita (descripción de imágenes o creación de historias), gramática (signos de puntuación, mayúsculas, género, nombre...) y vocabulario. En ciencias naturales los contenidos giran en torno a los animales y las plantas, y en matemáticas se trabajan conceptos básicos como los números, las sumas y restas y los problemas, siempre acordes a su nivel cognitivo.

El apartado Agenda se ha vinculado a Google Calendar, por lo que para acceder a su agenda personal la alumna necesita introducir su cuenta de correo electrónico de Gmail y la contraseña correspondiente. Con ella se pretende que la alumna se organice en el tiempo, apuntando los acontecimientos y eventos en la fecha adecuada.

Finalmente, en la sección Guías la usuaria puede encontrar diferentes enlaces con ayudas para desarrollar la autodeterminación, divididos en tareas del hogar, lugares públicos (cines, restaurantes, bibliotecas...) y recetas. En cada uno de ellos se dan instrucciones utilizando la metodología de lectura fácil; es decir, frases sencillas con las palabras clave resaltadas para facilitar su comprensión junto con otros apoyos visuales. 
Todos los contenidos de la web se pueden modificar a lo largo del proceso de intervención, lo que permite cambiar el nivel de dificultad de cualquier actividad o incluso añadir contenido. Esta es una de las ventajas del uso de tecnologías ágiles, pues permiten hacer modificaciones de forma sencilla y rápida, perfeccionando así el proceso de aprendizaje de la usuaria.

Una vez diseñada la intervención, se procedió a llevarla a cabo. En primer lugar, se enseñó a la alumna a trabajar con la página web y se le explicaron los motivos por los que era conveniente que lo hiciera. También se les explicó el funcionamiento a los padres para que cuando trabajara con la página web de forma autónoma en casa, ellos pudieran guiarle en caso de que fuera necesario.

La intervención con la participante consistió en 16 sesiones semanales que tuvieron lugar entre mediados de noviembre de 2019 y principios de marzo de 2020 . Se trabajó de forma individual con la alumna y siempre en su aula habitual de la fundación.

Cada sesión se dividía en una parte teórica-explicativa en la que se le proporcionaba una serie de instrucciones sobre el uso de la página web mediante ejemplos visuales y prácticos. Le seguía una parte autónoma, en la que la alumna navegaba de forma independiente a través de la herramienta TIC. En esta parte se evaluaba mediante observación directa la comprensión de las tareas por parte de la joven y si contaba con las competencias tecnológicas necesarias. Así, la alumna ponía en práctica las destrezas que posteriormente aplicaría individualmente en casa.

Al finalizar las sesiones, una vez cada dos semanas aproximadamente, tenían lugar las reuniones de seguimiento con la tutora y la familia, para informarles de las novedades o modificaciones incluidas en la herramienta y de los avances observados en la alumna.

Finalizada la intervención se pasó a la última fase del estudio, en la que se repitió la evaluación tanto a la estudiante como a sus padres utilizando la escala ARC-INICO (Verdugo et al., 2014) para comparar los resultados pretest y posttest y así valorar la efectividad de la intervención y la consecución de los objetivos planteados. El procedimiento en la cumplimentación del cuestionario por ambas partes fue exactamente igual que en la primera ocasión.

\section{Análisis de datos}

Para el tratamiento estadístico de los datos se utilizaron los baremos proporcionados por el manual de la escala ARC-INICO (Verdugo et al., 2014), y el procedimiento fue el mismo tanto para los obtenidos en la evaluación de la estudiante como en la de sus familiares.

En primer lugar, se efectuó el cálculo de las puntuaciones directas y estandarizadas para cada una de las cuatro dimensiones que evalúa la escala, en el pretest y el posttest. A continuación, para calcular la puntuación global de autodeterminación se sumaron las puntuaciones directas, obteniendo una puntuación total que, en base al baremo del cuestionario, se convirtió en puntuación estandarizada global y se situó en el percentil correspondiente.

Tal y como se indica en el manual de la escala (Verdugo et al., 2014), se debe considerar que las puntuaciones estandarizadas presentan una distribución con media igual a 100 y una desviación típica igual a 15. De este modo, para la correcta interpretación de los resultados, es necesario transformar las puntuaciones directas en puntuaciones estandarizadas. Cuanto mayor es la puntuación obtenida, mayor es el nivel de 
autodeterminación de la persona evaluada. Junto a la puntuación estandarizada, cada baremo proporciona un percentil correspondiente, esto indica el porcentaje de personas que tienen una puntuación inferior a la de la persona evaluada. Por tanto, cuanto mayor es el percentil obtenido, mayor será el nivel global de autodeterminación.

Los resultados de la escala ARC-INICO proporcionan información precisa sobre el nivel global de autodeterminación de la persona evaluada pero también sobre dimensiones concretas, pudiendo conocer así en cuáles presenta más fortalezas y en cuáles más debilidades.

\section{Resultados}

En las tablas 1 y 2 se reflejan los resultados obtenidos en la evaluación de la estudiante antes y después de la intervención. Destaca principalmente la dimensión de autonomía por la mejora producida tras la intervención, ya que en el percentil se encuentra una diferencia de 54 puntos entre el pretest y posttest. Respecto a los resultados globales, es resaltable el aumento de 14 puntos en el percentil de la puntuación estandarizada global (PEG) tras la intervención.

Tabla 1. Puntuaciones de los cuestionarios de la alumna.

\begin{tabular}{lllllll}
\hline & \multicolumn{2}{l}{ Puntuación directa } & Puntuación estandarizada & \multicolumn{2}{l}{ Percentiles } \\
\hline & Pretest & Posttest & Pretest & Posttest & Pretest & Posttest \\
\hline Autonomía & 35 & 54 & 72 & 102 & 2 & 56 \\
Autorregulación & 19 & 25 & 72 & 79 & 1 & 6 \\
Empoderamiento & 24 & 33 & 67 & 79 & 1 & 7 \\
Autoconocimiento & 26 & 29 & 81 & 92 & 12 & 30 \\
\hline
\end{tabular}

Tabla 2. Sumatorios de las puntuaciones de la alumna.

\begin{tabular}{ccc}
\hline & Pretest & Posttest \\
\hline Sumatoria puntuación & 104 & 141 \\
Puntuación estandarizada global & 67 & 82 \\
Percentil PEG & 0 & 14 \\
\hline
\end{tabular}

En las tablas 3 y 4 se exponen los resultados obtenidos con las respuestas de los padres. La dimensión en que se ha adquirido mayor nivel es, igual que en los resultados anteriores, la autonomía, con una diferencia de 47 puntos de percentil. Los de nivel menor siguen siendo la autorregulación y el empoderamiento, aunque con mayor aumento, de 20 y 18 puntos respectivamente. Asimismo, es satisfactorio el incremento en el percentil PEG en la evaluación posttest, ya que alcanza el valor de 27 puntos.

Tabla 3. Puntuaciones de los cuestionarios de los padres.

\begin{tabular}{lllllll}
\hline & \multicolumn{2}{l}{ Puntuación directa } & \multicolumn{2}{l}{ Puntuación estandarizada } & \multicolumn{2}{l}{ Percentiles } \\
\hline & Pretest & Posttest & Pretest & Posttest & Pretest & Posttest \\
\hline Autonomía & 37 & 53 & 75 & 100 & 4 & 51 \\
Autorregulación & 22 & 30 & 75 & 88 & 2 & 22 \\
Empoderamiento & 26 & 37 & 67 & 84 & 1 & 19 \\
Autoconocimiento & 27 & 31 & 84 & 99 & 17 & 46 \\
\hline
\end{tabular}


Tabla 4. Sumatorios de las puntuaciones de los padres.

\begin{tabular}{ccc}
\hline & & \\
\hline Sumatorio puntuación & Pretest & Posttest \\
\hline Puntuación estandarizada global & 112 & 151 \\
Percentil PEG & 69 & 91 \\
& 1 & 28
\end{tabular}

En general, tanto en los resultados obtenidos en la evaluación pretest y posttest de la estudiante como en la de sus padres, se aprecia una influencia positiva de la intervención en el nivel de autodeterminación de la participante.

Las entrevistas con la tutora y la familia a lo largo de toda la intervención fueron de gran utilidad para complementar los datos sistemáticos obtenidos a través del cuestionario. Resulta importante contrastar los resultados a través de herramientas de evaluación estandarizadas con la propia experiencia de los participantes. El feedback proporcionado a través de estas entrevistas apuntala los resultados ofrecidos a través de las puntuaciones de la escala, ya que tanto la tutora como la familia corroboran que, tras la intervención, la estudiante mejoró su capacidad para organizar su propio tiempo y aumentó sus niveles de autonomía en tareas como la realización de actividades académicas.

\section{Discusión}

El objetivo del presente estudio era diseñar, implementar y evaluar una intervención con una herramienta TIC con objeto de mejorar la autodeterminación de una alumna con síndrome de Down. La valoración general, tras llevar a cabo la intervención, es que se ha producido una mejora de las dimensiones de autodeterminación analizadas en la alumna en unos niveles razonables, dada la duración de la intervención.

Los resultados obtenidos en la evaluación pretest del nivel de autodeterminación de la alumna mostraron que el ámbito con mejores resultados era el autoconocimiento, aunque desde el punto de vista de los padres la dimensión en que su hija presentaba mayor nivel era el autoconcepto. En los ámbitos de autorregulación y empoderamiento la puntuación fue excesivamente baja en la evaluación pretest tanto de la alumna como de su familia.

Tras la aplicación del programa de intervención, el ámbito en el que la alumna muestra una mejora notable es la autonomía, mientras que en la autorregulación y el empoderamiento, pese a que se obtiene una puntuación más alta, sigue mostrando un nivel bajo. La percepción de la familia es la misma.

Estos hallazgos se pueden atribuir probablemente al carácter autónomo de la herramienta TIC diseñada. Aunque abarca diferentes dimensiones de la autodeterminación, la autonomía es la más reforzada ya que todas las posibilidades que ofrece la página web se dirigen a una resolución y un manejo individual y autónomo por parte de la usuaria.

Así pues, comparando las puntuaciones obtenidas en cada una de las secciones de la escala, antes de la intervención y al terminar la misma, se confirma una mejora global de la autodeterminación de la estudiante, especialmente en el ámbito de autonomía. Estos buenos resultados podrían atribuirse, además de a las características de la herramienta TIC, a la implicación y participación constante de la alumna y su familia durante todo el proceso. 
Los resultados de este estudio se alinean con los de investigaciones previas en las que se ha analizado si el uso de las TIC repercute positivamente en la calidad de vida de las personas con discapacidad. Cullen y Alber-Morgan (2015) hallaron en su revisión teórica que en diversos estudios la tecnología había conllevado a un aumento de la independencia de algunos usuarios con discapacidad intelectual para realizar actividades cotidianas. Esto mismo se ha hallado en este estudio al apreciar un notorio incremento en la autonomía de la participante tras trabajar con una herramienta TIC.

Los estudios de Cannella-Malone et al. (2015) o Stasolla et al. (2014) son otro ejemplo de investigaciones empíricas como esta en que, tras el uso de determinadas herramientas tecnológicas, se encuentran beneficios en el desarrollo personal de los usuarios con diversidad funcional.

Por tanto, aunque este campo de estudio sea todavía reducido, las evidencias que existen hasta ahora confirman que las TIC son herramientas valiosas para las personas con discapacidad, lo que sugiere la conveniencia de continuar llevando a cabo nuevas investigaciones que respalden con nuevos resultados empíricos la afirmación de que las TIC pueden contribuir a la mejora de la autodeterminación de las personas con discapacidad.

El conjunto de estos resultados conllevaría implicaciones prácticas en, al menos, dos ámbitos diferenciados.

La primera implicación sería la necesidad de orientar la formación inicial y continua del profesorado hacia una capacitación en el manejo de las TIC que permita aprovechar estas potencialidades. Las investigaciones que han analizado la percepción del propio profesorado para emplear las TIC para la mejora de la educación de estudiantes con NEE arrojan resultados pesimistas (Fernández, Román y Siles, 2020; Llorente y Toledo, 2016). Estos resultados sugieren la necesidad de repensar el modelo de formación del profesorado y orientarlo para que verdaderamente pueda capacitarse a los futuros profesionales de la educación y dotarles de una competencia digital docente que permita aprovechar en la práctica la potencialidad de los recursos TIC para la educación de los estudiantes con NEE.

La segunda implicación está relacionada con las posibilidades reales de implementación de recursos TIC en las aulas. Aunque la mayoría de los centros dispongan de una cantidad y calidad razonable de recursos TIC, no siempre se cuenta con estrategias organizativas que permitan su uso de una manera integrada en el currículum (Pettersson, 2018). Por ello, es necesario un cambio en la manera en que se conceptualizan las TIC en nuestro sistema educativo, de manera que se dejen de emplear las TIC como un objetivo en sí mismo, y se tienda más a pensar en los recursos TIC como un medio para alcanzar determinados objetivos, como en este caso el aumento de la capacidad de autodeterminación en una estudiante con discapacidad intelectual.

Los resultados obtenidos en este estudio y las conclusiones extraídas a partir de ellos deben entenderse teniendo en cuenta, al menos, tres limitaciones que conllevan asimismo implicaciones para futuras investigaciones.

La primera de ellas es la duración de la intervención (16 sesiones distribuidas en cerca de 4 meses). Aunque se trata de un período de tiempo razonable, no permite conocer si los beneficios obtenidos continuarían incrementándose en caso de extender la duración a todo el curso, o bien si se observaría un efecto meseta o incluso de retroceso en la evolución de los resultados. Probablemente, uno de los factores que ha influido en 
la obtención de resultados positivos en la presente intervención ha sido el de que se trataba de una intervención nueva, diferente a las que se habían desarrollado anteriormente, lo que ha podido tener un impacto en la motivación de la estudiante. Es esperable que esta mejora en la motivación pueda decaer cuando se atenúe el efecto novedad, por lo que sería interesante llevar a cabo intervenciones de un curso completo de duración que permitan analizar a más largo plazo los resultados de la implementación de las intervenciones basadas en las TIC.

La segunda limitación es el tamaño de la muestra. Al tratarse de un solo sujeto no es posible hacer generalizaciones significativas a partir de los datos obtenidos, por lo que sería interesante en el futuro replicar esta intervención con muestras más amplias de participantes, que además presentaran diferentes características (edad, severidad de la discapacidad intelectual y comorbilidades asociadas), para así analizar si la implementación de este tipo de intervenciones es especialmente efectiva en un segmento determinado de edad o en un subgrupo de personas con discapacidad intelectual.

La tercera limitación está relacionada con los instrumentos de evaluación. Es posible que al utilizar un instrumento de autoinforme los resultados obtenidos estén influidos por el sesgo de deseabilidad social, de manera que las respuestas se emitan no únicamente por la valoración sincera de los usuarios, sino también por lo que éstos consideran que se espera que deberían responder. Por ello, sería conveniente en futuros estudios complementar la metodología con entrevistas que permitan la triangulación de resultados provenientes de diferentes fuentes.

La finalización del presente estudio abre la puerta a futuras líneas de investigación que podrían conducir a nuevos resultados interesantes.

Una primera futura vía de investigación consistiría en formar e implicar a las familias en la supervisión de las intervenciones basadas en TIC. El uso de las TIC por parte de niños y adolescentes no está exento de riesgos, especialmente en el caso de personas con discapacidad (Chiner, Gómez-Puerta y Cardona-Moltó, 2017; Gómez-Puerta y Chiner, 2019). Por ello es necesario que las familias ejerzan un rol relevante en la supervisión de su uso y que estén suficientemente formados en el conocimiento de los riesgos que implican las TIC.

Finalmente, otra posible vía de investigación consistiría en plantear diseños que comparen los efectos de intervenciones en que las TIC tengan diferentes grados de protagonismo, de manera que se combinen con intervenciones sin componentes TIC. De este modo, podríamos obtener información acerca de qué papel juegan exactamente los recursos TIC en la mejora de la autodeterminación y qué aportan verdaderamente estos recursos a las intervenciones que no incluyen este tipo de recursos.

\section{Referencias}

American Psychiatric Association (2013). Diagnostic and statistical manual of mental disorders (5th ed.). Arlington, VA: American Psychiatric Publishing.

Cannella-Malone, HI, Konrad, M, \& Pennington, RC (2015). ACCESS! Teaching writing skills to students with intellectual disability. Teaching Exceptional Children, 47(5), 272-280. 
Chiner, E, Gómez-Puerta, M, \& Cardona-Moltó, MC (2017). Internet use, risks and online behaviour: The view of internet users with intellectual disabilities and their caregivers. British Journal of Learning Disabilities, 45(3), 190-197.

Claes, C, Van Hove, G, Vandevelde, S, Van Loon, J, \& Schalock, R (2012). The influence of supports strategies, environmental factors, and client characteristics on quality of life-related personal outcomes. Research in Developmental disabilities, 33(1), 96-103.

Cullen, JM, \& Alber-Morgan, SR (2015). Technology mediated self-prompting of daily living skills for adolescents and adults with disabilities: A review of the literature. Education and Training in Autism and Developmental Disabilities, 50(1), 43-55.

Draper, C, Strnadová, I, \& Cumming, T (2014). Using iPads with students with disabilities: Lessons learned from students, teachers, and parents. Intervention in School and Clinic, 49(4), 244-250.

Fernández, A (2016). Aspectos generales sobre el síndrome de Down. Revista Internacional de apoyo a la inclusión, logopedia, sociedad y multiculturalidad, 2(1), 33-38.

Fernández, JM, Reyes, MM y El Homrani, M (2018). TIC y discapacidad. Principales barreras para la formación del profesorado. EDMETIC, Revista de Educación Mediática y TIC, 7(1), 1-25.

Fernández, JM, Román, P, \& Siles, C (2020). Are primary education teachers from Catalonia (Spain) trained on the ICT and disability?. Digital Education Review, 37, 288-303.

Gómez-Puerta, M, \& Chiner, E (2019). Teachers' perceptions on online behaviour of students with intellectual disability, risk mediation and training. European Journal of Special Needs Education.

Llorente, M, \& Toledo, P (2016). Initial teacher training in the use of information and communications technology (ICT) for education of the disabled. Digital Education Review, 30, 135-146.

Peralta, F y Arellano, A (2014). La autodeterminación de las personas con discapacidad intelectual: situación actual en España. Revista CES Psicología, 7(2), 59-77.

Pettersson, $F$ (2018). On the issues of digital competence in educational contexts - a review of literature. Education and Information Technologies, 23, 1005-1021.

Stasolla, F, Damiani, R, Perilli, V, Di Leone, A, Albano, V, Stella, A, \& Damato, C (2014). Technological supports to promote choice opportunities by two children with fragile $X$ syndrome and severe to profound developmental disabilities. Research in Developmental Disabilities, 35(11), 2993-3000.

Verdugo, MA, Vicente, E, Gómez-Vela, M, Fernández, R, Wehmeyer, M, Badia, M., González, F y Calvo, MI (2014). Escala ARC-INICO de Evaluación de la Autodeterminación. Manual de aplicación y corrección. Salamanca: Inico. 